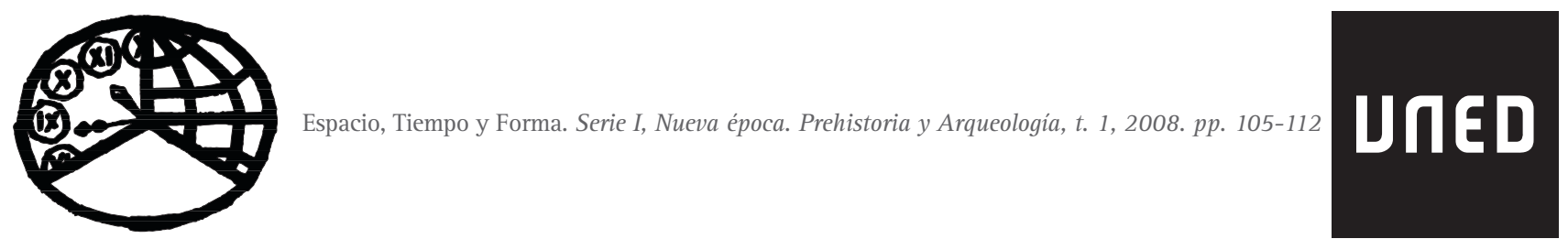

\title{
LA IMAGEN EN EL ARTE DE LAS CUEVAS DEL MONTE DEL CASTILLO
}

\author{
L'image dans l'art des grottes du Mont Castillo
}

\author{
Marc Groenen*
}

Recibido 17 de septiembre de 2007. Aceptado el 30 de octubre de 2007.

\begin{abstract}
Resumen. Los artistas paleolíticos poseían una serie de técnicas extraordinariamente rica, pero también un saber hacer experimentado. Constatamos que los motivos figurativos imponen al espectador su unidad y su coherencia formal. Éstos nunca parecen como la mera adición de trazos independientes, pero cada uno de ellos se da, al contrario, desde el resultado final. Mejor, la figura animal nunca es desencarnada: el artista le da muy a menudo su forma, su volumen y su realidad, integrando particularidades naturales o utilizando procedimientos gráficos sugestivos. Pues, el arte parietal es mucho más un arte de la sugestión que un arte de la ilusión. Nos parece entonces cierto y que el arte rupestre es la obra de profesionales. Palabras clave: Arte paleolítico, arte rupestre, imagen, artistas paleolíticos, sistema estético.
\end{abstract}

Resumé. Les artistes paléolithiques possédaient une série de techniques très riche, ainsi qu'un savoir-faire expérimenté. Nous constatons que les motifs figuratifs imposent leur unité et leur cohérence formelles au spectateur. Ceux-ci n'apparaissent jamais comme la simple addition de traits indépendants, mais chacun d'entre eux se donnent, au contraire, depuis le résultat final. Mieux encore, la figure animale n'est jamais désincarnée : l'artiste lui donne bien souvent sa forme, son volume et sa réalité en intégrant des particularités naturelles ou en utilisant des procédés graphiques suggestifs. L'art pariétal est donc beaucoup plus un art de la suggestion qu'un art de l'illusion. II nous semble dès lors assuré que l'art pariétal est l'oeuvre de professionnels. Mots-clés: Art paléolithique, art pariétal, image, artistes paléolithiques, système esthétique.

Cada uno sabe que el hombre del Paleolítico superior (dentro de 35.000 y 9.000 a. C.) utilizó las cuevas para decorar las paredes. La cueva de Altamira, descubierta por Marcelino Sainz de Sautuola, es famosa en cuanto a esto, como docenas de otras en España y en Francia. El arte prehistórico es hoy bien conocido y comprende miles de figuras.

Sin pensar como, utilizamos todos, como yo mismo, el término "arte" para calificar los motivos estéticos de este periodo. Sin embargo, nadie pensaría relacionar o comparar las imágenes de bisontes, de caballos o de ciervas de cuevas decoradas con los personajes de las pinturas de Velázquez, por ejemplo. Sin darnos cuenta de eso, hacemos una distinción clara entre las representaciones prehistóricas y las re- presentaciones de las épocas históricas. Los pintores o los escultores de los periodos históricos son efectivamente considerados como artistas profesionales. Recibieron una formación, gracias a la cual aprendieron todas las sutilidades de las técnicas de dibujo y de pintura. $Y$ este aprendizaje complejo les permitió representar personajes, animales u objetos con una exactitud extraordinaria. Tal es la "personalidad artística" de esos grandes artistas de las épocas históricas que un especialista de la historia del arte puede identificar la época, la región y el autor, incluso cuando la pintura no está firmada. El análisis estilístico permite caracterizar la época del pintor, y la atribución permite a veces precisar el pintor mismo.

(*) Profesor de Arte y Arqueología prehistóricos a la Universidad Libre de Bruselas (U.L.B.), C.P. 175, Servicio de los Civilizaciones Primeras, Avenida F.D. Roosevelt 50, B-1050 Bruselas, Bélgica - mgroenen@ulb.ac.be. 
¿Pero, cuál es la situación para el hombre de Cro-Magnon que estudiamos? Constatamos la calidad gráfica de las pinturas y de los grabados rupestres espléndidos que hizo. Vemos la exactitud y la precisión del trazado, hasta tal punto que podemos fácilmente identificar el animal representado, e incluso su comportamiento. Sin embargo, la representación mental que tenemos de este hombre nos impide considerarlo como un verdadero artista. Lo vemos sobre todo como un cazador, intentando sobrevivir en una naturaleza ingrata, con instrumentos muy primitivos. Imaginamos que dedicaba toda su energía a alimentarse, a protegerse del frío y de los animales peligrosos. Naturalmente, es imposible conciliar la idea de un pobre salvaje errando en una naturaleza hostil, con la de un artista realizando su talento artístico. $Y$, sin embargo, nos quedamos con esta contradicción: los espléndidos bisontes policromos de Altamira fueron dibujados, pintados y grabados por salvajes vestidos con pieles de animales, intentando, como podian, representar dichos animales, frotando y escupiendo el color en el techo de una sala oscura, gruñendo para que sus compañeros les entendiesen. Esta imagen del hombre es totalmente absurda. Sobre todo, es incompatible con lo que nos enseñan las obras que realizó.

Como en el caso del arte "arcaico" de África, de América del Sur o de Oceanía, los artes prehistóricos nunca han sido considerados desde el punto de vista de la historia del arte. Los prehistoriadores se esmeran, y es normal, en identificar los motivos, en datarlos, en describir su situación en la cueva, pero nunca en tratar la imagen misma. En cuanto a los trabajos publicados por los historiadores del arte, es decir los profesionales del análisis de la imagen, ignoran muy a menudo lo que es anterior al arte de la Antigüedad, es decir el arte de Egipto o de Grecia.
Es precisamente el punto de vista del historiador del arte que me gustaría utilizar. Quisiera, particularmente, intentar mostrar que los hombres de esta antigua época no se contentaron con trazar figuras sin un perfecto dominio de la técnica y del grafismo. El análisis riguroso de los motivos parietales revela que estas figuras son el producto de pintores o grabadores experimentados. Estos debían tener un saber hacer extraordinario, que sólo pueden tener artistas que recibieron una verdadera formación. Eso explica y justifica sistemas estéticos notablemente coherentes, pero también personalidades estéticas claramente distintas. Nuestros ejemplos vendrán de las cuevas decoradas del Monte del Castillo (Puente Viesgo), en Cantabria.

\section{DOMINIO TÉCNICO Y RESULTADO ÓPTICO}

Sabemos que los hombres prehistóricos consiguieron con mucha habilidad obtener un trazado perfecto, a pesar de las irregularidades del soporte. Ni las fisuras, ni los sobresalientes de la roca, ni la calcita les impidieron realizar sus motivos. Sin embargo, en ciertos casos, estos hombres acondicionaron la pared o, más exactamente, la prepararon. El hecho era bien conocido en la cueva de Altxerri (Altuna, 1997), pero no había sido subrayado en las cuevas decoradas cantábricas. En una de las salas de la cueva del Castillo, dos bisontes muy hermosos fueron trazados en negro sobre una cornisa. El pequeño bisonte, probablemente un ternero, ubicado en la izquierda, fue ejecutado en una parte previamente frotada de la pared. Si miramos bien, vemos efectivamente que la pared es más blanca en la figura y alrededor. Si miramos aún con más atención, vemos también que esa zona blanquecina desborda un poco el trazado negro de la figura (fig. 1). Se trata

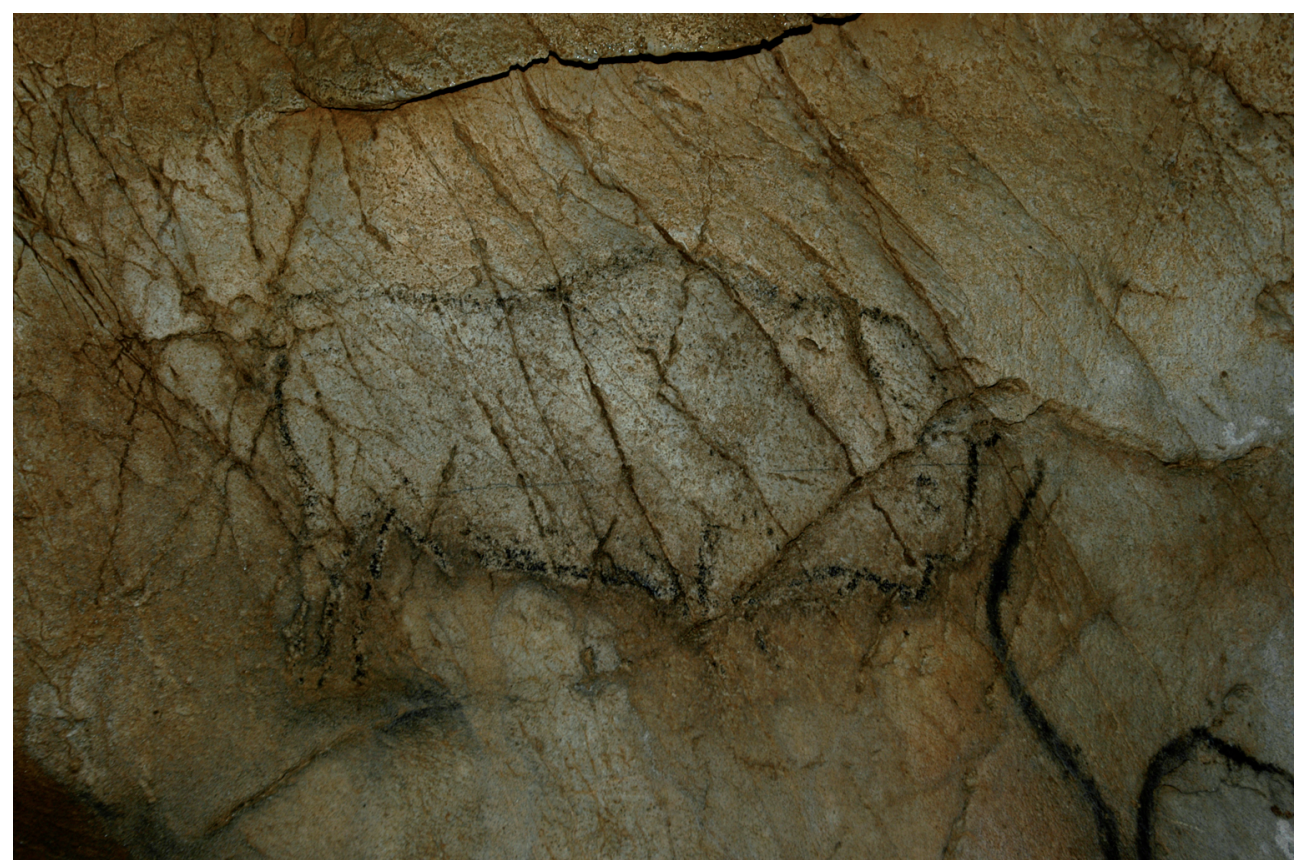

- Figura 1. Cueva del Castillo, Sala B: ternero negro ubicado en una cornisa. 
de una preparación del soporte que permite a la figura que resalte, que se destaque mejor del soporte. Tal preparación, muy cuidadosa, ha sido también evidenciada en otros lugares de la cueva, y en particular en el pequeño uro negro del "Divertículo de la Gran Sala" (Alcalde del Río et al., 1912: 145, fig. 134, n.os 3 y 6). Una vez más, un rascado muy fino y quizás una limpieza de la pared destinada a aclararla se produjeron antes de trazar el pequeño animal.

Estamos a menudo impresionados por la calidad gráfica de las pinturas o de los dibujos. Casi nunca se ve una hesitación, un repintado, un trazado "malogrado". Ahora bien, es difícil borrar completamente un trazado en la roca: siempre queda un resto débil. Si nada es visible, tenemos que preguntarnos como los artistas del Paleolítico superior consiguieron tal precisión en la realización de sus obras. En el arte de los periodos históricos, los pintores hicieron un trazado preparatorio, que se puede evidenciar por técnicas de laboratorio como el infrarrojo o la radiografía. En el arte prehistórico, donde esas técnicas no han podido ser probadas, es difícil demostrar la existencia de esa práctica. Efectivamente, o el trazado definitivo quitó el trazado preparatorio, si se trata de un grabado, o lo tapó, si se trata de una pintura. Unos bisontes negros de la cueva de Niaux, en Francia, por ejemplo, fueron primero bosquejados con carbón vegetal, antes de su ejecución con negro de manganeso (Clottes et al., 1990: 179-181). La realización de un trazado preparatorio previamente al dibujo definitivo se pudo demostrar en El Castillo también. Una línea cervico-dorsal de cierva del primer panel fue primero realizada con un trazado continuo muy fino, de color rojo violáceo, y después por pequeñas puntuaciones yuxtapuestas. Las técnicas son diferentes para los dos trazados, como lo son los matices de color. Sobre todo, el trazado continuo pasa debajo de las puntuaciones, lo que permite interpretarlo como un trazado preparatorio (Groenen, 2007a; 2007b). Nuestras investigaciones continúan en esta dirección para otras figuras, porque la existencia de un trazado anterior al poner en limpio es un hecho particularmente importante para nuestra comprensión del proceso artístico. Muestra en efecto la voluntad de una realización "diferida" de la figura. El esbozo permite al ejecutante construir su figura, haciendo coincidir lo más precisamente posible su trazado con el proyecto estético que concibió. Mientras que la producción inmediata le da enseguida a la obra un carácter definitivo e irrevocable, el trabajo preparatorio permite al ejecutante hacer cristalizar en su realización una investigación siempre en curso al momento de la elaboración.

Los trabajos dedicados al arte parietal insistieron suficientemente sobre la multiplicidad de técnicas utilizadas por los hombres del Paleolítico: pinturas, dibujos, grabados o relieves siempre han sido manipulados con facilidad y tacto. No obstante, aunque parezcan claros, esos términos son demasiado generales. En efecto, no dan cuenta de la riqueza de los procedimientos utilizados o de la complejidad de las

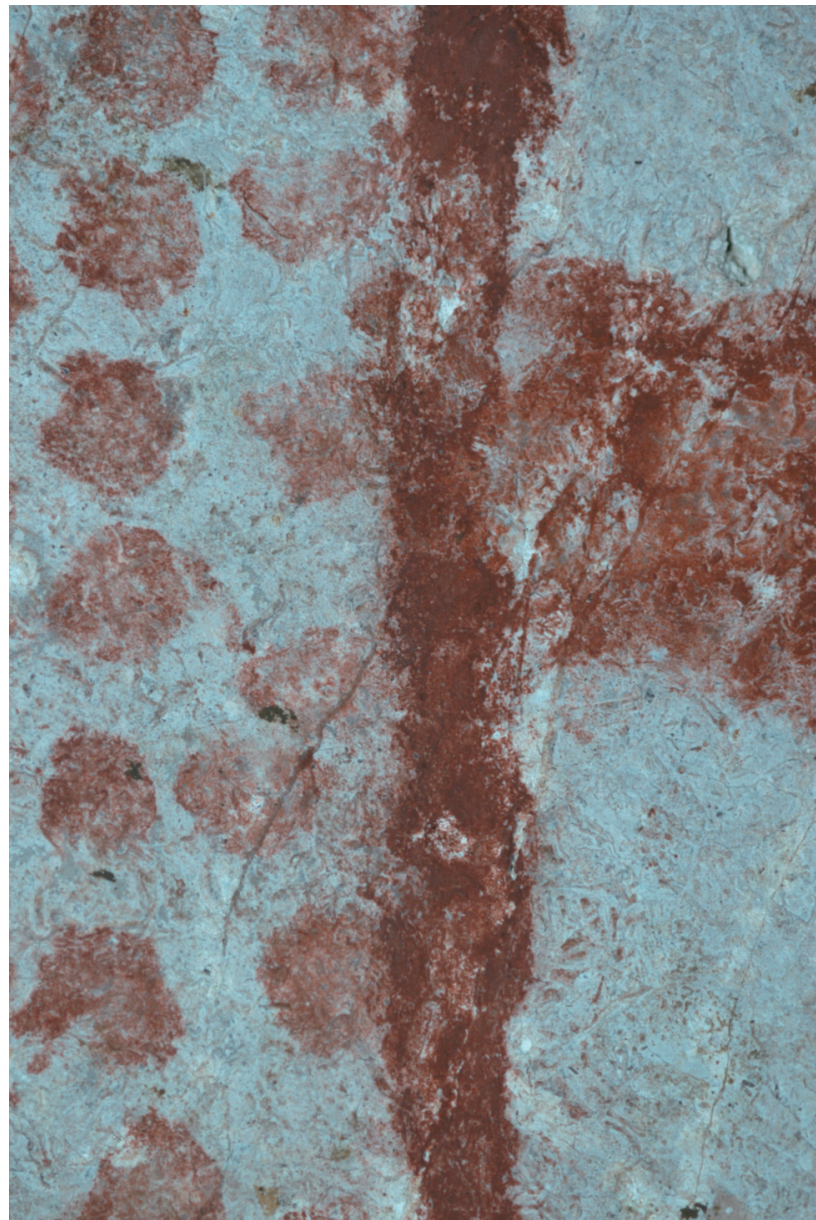

A Figura 2. Cueva del Castillo, Rincón de los Tectiformes: puntos rojos unidos por un trazo de color.

cadenas operatorias puestas en acción. Si las preciosas figuras de ciervas rojas "punteadas" de La Pasiega o de Covalanas, por ejemplo, son bien conocidas, se sabe menos que numerosas figuras de las cuevas cantábricas fueron realizadas con la misma técnica. Pero fueron hechas con tal cuidado que no es siempre posible ver los pequeños puntos de color formando el contorno del animal o del "signo" sin ampliaciones fotográficas. Por esta razón, han sido realizadas sistemáticamente por un trazado continuo. Un mamut del Castillo (Alcalde del Río et al., 1912: 129, fig. 117, n. 76 , pl. LXXIII), por ejemplo, está totalmente formado de pequeños puntos de color, así como una cabeza de uro (Alcalde del Río et al.: 128, fig. 117 n. 3 , pl. LXXVIII, n.o 1) y una línea cervico-dorsal de cierva de la misma cueva (Alcalde del Río et al., 1912: fig. 122, n. ${ }^{\circ}$ 17, pl. LXXI). Pues, el contorno no está constituido por un trazado, sino por unas series de puntos a menudo muy cercanos, hasta tal punto que no se pueden individualizar.

Esta misma técnica también fue empleada para pintar los signos cuadrangulares compartimentados de la cueva del Castillo, en particular en el famoso "Rincón de los Tectifor- 
mes", pero con puntuaciones más anchas. Un examen cuidadoso muestra que el contorno fue de primeras realizado con puntos dispuestos los unos al lado de los otros. Luego, esos puntos fueron reunidos con un trazo de color (fig. 2). Pues, la técnica de base es la misma que la que fue utilizada para las series de puntos del mismo lugar de la cueva. ¿Pero cómo se realizaron esos puntos? Podríamos pensar que fueron realizados con un dedo directamente empapado de pintura. No es el caso, porque la parte central del disco a menudo no muestra color. Ahora bien, la huella del dedo siempre es más densa en el centro. Además, el contorno externo presenta un borde finamente acentuado. Esos elementos nos permiten pensar que los puntos fueron hechos con un instrumento hueco o cóncavo, probablemente cubierto de una piel fina. Así, no se trata de meros trazados, rápidamente hechos. Además, los motivos no fueron todos realizados con pinturas de tipo idéntico. Sin abordar la cuestión difícil de las cargas, que sólo los análisis pueden evidenciar (Clottes et al., 1990), unas experimentaciones indican sin duda alguna la utilización de materias colorantes de espesor variable. Unos resultados cercanos, en todo caso, han sido obtenidos con pinturas cuyas argamasa era grasa o tuétano (Richard, 2005-2006).

Se puede lógicamente también demostrar una virtuosidad de nivel técnico igual para los grabados. Una vez más, el vocabulario empleado por los prehistoriadores no da cuenta ni de la variedad de los instrumentos utilizados, ni de la complejidad de las cadenas operatorias puestas en acción. M. Crémadès $(1992,1996)$ primero y C. Fritz (1999) segundo lo mostraron bien para el arte mueble del suroeste francés. La realización de un animal sobre un fragmento de asta de reno o de hueso necesitó, en ciertos casos, la utilización sucesiva de seis técnicas distintas. En efecto, el artista adapta el ins- trumento y el ademán en función del efecto óptico que quiere producir. Un trazado cuya sección forma una " $U$ " simétrica, por ejemplo, aísla más el motivo que un trazado en "V" simétrica. Del mismo modo, el trazado de sección asimétrica le da al grabado unos efectos de profundidad. Se leerá luego más en términos de escultura que en términos de grabado.

Nuestros trabajos en las cuevas cantábricas, y en particular en El Castillo, nos indican el mismo alto nivel de dominio técnico. La incisión nítida y profunda de los motivos situados en el techo de la "entrada gravettiense" de dicha cavidad aíslan las figuras del soporte por un ribete de sombra producido por la luz. El caballo (Alcalde del Río et al., 1912: fig. 152, no. 1, pl. LXII, no. 4), por ejemplo, adquiere una autonomía sorprendente con respecto a la superficie sobre la cual ha sido hecho. Esta desolidarización produce un asombroso efecto de surgimiento de la figura, aún más acentuado por el bordo natural del medallón en el cual ha sido grabado. El resultado óptico es totalmente diferente en el caso de las ciervas rascadas de la "Sala A". Aunque es muy a menudo poco profundo, el trazado del contorno del animal está ligeramente acentuado en relación con las estrías internas, sea por un surco más ancho, sea por trazados repetidos. Las estrías internas a menudo son paralelas entre sí y proceden de un ademán seguro. Poco profundas, presentan casi siempre una sección en "V" simétrica, lo que le da a la figura una apariencia angulosa. Se inscribe harmoniosamente en el campo del motivo, dando a los segmentos anatómicos un modelado sugestivo. Podemos pensar que, en esta época, esas figuras animales debían presentarse mucho más sobre la forma de motivos colorados que sobre la forma de grabados. En efecto, la eliminación de la patina superficial debía acentuar el color blanco de la calcita o la coloración gris-azul de la roca.

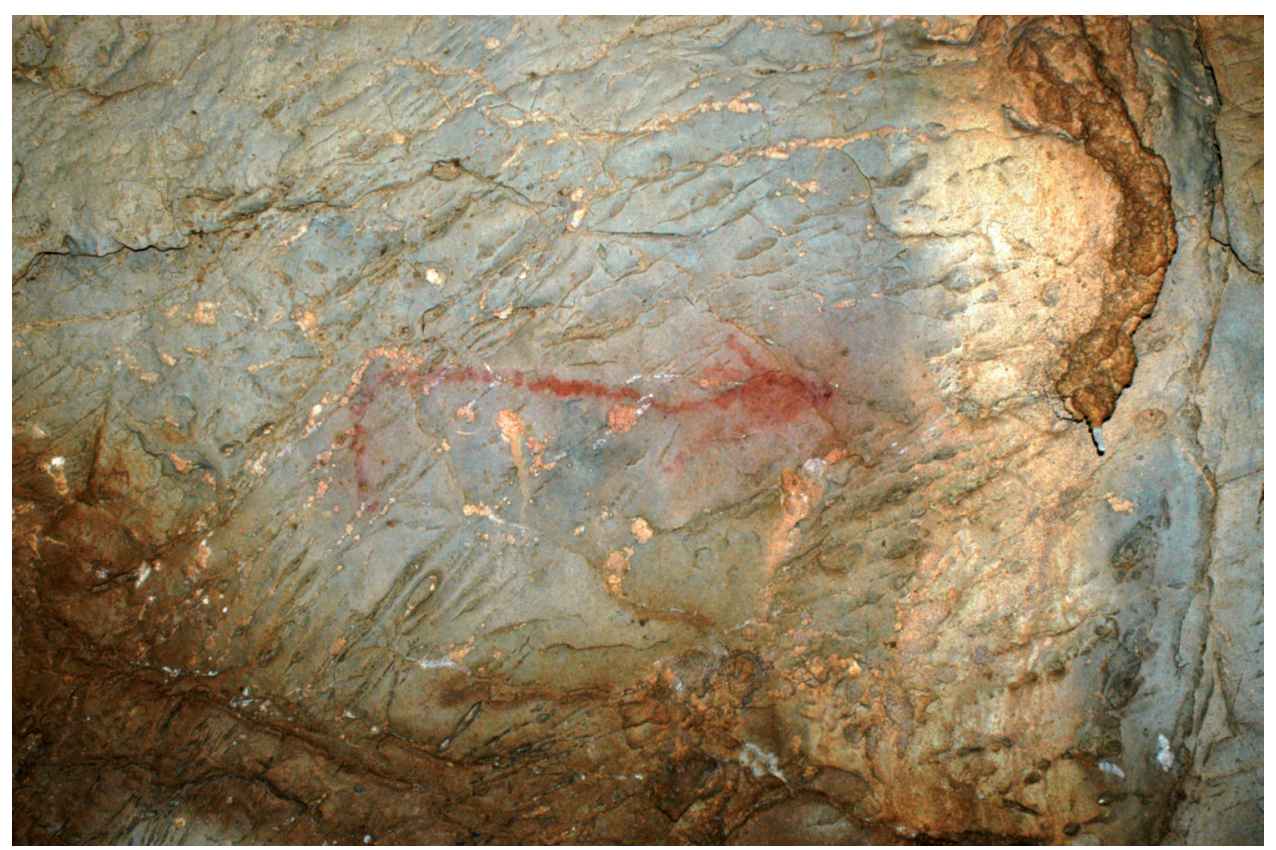

- Figura 3. Cueva de La Pasiega A: cierva roja (trazado rígido o irregular). 


\section{DOMINIO GRÁFICO}

Si el dominio técnico se impone, la extraordinaria calidad gráfica de las obras tiene que retener también toda nuestra atención. A pesar de las dificultades planteadas por las irregularidades del soporte rocoso, los artistas no tuvieron aparentemente ninguna dificultad en representar animales casi siempre perfectamente identificables. Por tanto, no deberíamos pensar que el animal representado fuese una mera copia de la naturaleza. Hay una elección en los detalles figurados, y ciertas partes anatómicas están representadas en función de convenciones estilísticas estrictamente codificadas. Así, el animal está más a menudo sistemáticamente figurado de perfil, con excepción de ciertas partes, como las pezuñas, las astas o los cuernos, que están a menudo presentados de frente. Estos detalles son evidentemente importantes, porque nos permiten muy frecuentemente precisar la identificación. Así pues, es normal presentarlos de frente. Si se nota una semejanza estilística muy general, por lo tanto todas las figuras no son idénticas. En efecto, podemos muy a menudo distinguir grupos estilísticos muy diferentes, e incluso en una misma red. Algunos ejemplos de La Pasiega A lo demuestran. Notaremos de primero que hay artistas que son evidentemente menos dotados que otros: realizan figuras más esquemáticas, con un trazado rígido o irregular (fig. 3). Otros, al contrario, construyen sus figuras iniciando curvas grandes, sobre las cuales se insertan los otros segmentos (fig. 4). Y otros hacen trazados muy flexibles, con curvas y contra-curvas, con un resultado mucho más orgánico (fig. 5). Es inútil multiplicar los ejemplos: cuando miramos con atención las obras del arte parietal, parece que tienen cada una su personalidad estilística. Por eso, es difícil atribuir todas las obras de una cueva a un estilo úni- co, como lo había hecho Leroi-Gourhan $(1965,19782)$ en otro tiempo.

Tampoco deberíamos pensar que cada animal fue hecho por un ejecutante diferente, como lo habían imaginado Henri Breuil, Hugo Obermaier o Henri Bégouën, en el marco de su teoría de la magia de la caza (Groenen, 1994). Parece al contrario que sea posible que diferentes animales fuesen realizados por un solo artista, como el método de la autoría lo permite demostrar. Este consiste en localizar costumbres gráficas muy particulares. Cuando un artista hace un dibujo o una pintura, lo hace en función de esquemas aprendidos que repetirá. Así, algunos detalles del trazado presentan un carácter muy personal, un poco como la escritura de cada uno de nosotros tiene su personalidad. Este tipo de análisis permitió, desde hace mucho tiempo, a los historiadores del arte identificar artistas de pinturas no firmadas. Se puede también aplicar al arte paleolítico. Hemos mostrado en otro lugar (Groenen y Martens, en prensa) que seis figuraciones de la cueva de La Pasiega $A$, ubicadas en diferentes lugares de la cueva, pueden estar atribuidas a un solo y mismo artista. Dentro de estas obras, notamos una cierva, dos caballos, dos uros y un rebeco. Todos estos animales comparten caracteristicas comunes, dentro de las cuales algunas son demasiado particulares para que no fuesen imputadas a un solo artista. Sin entrar demasiado en detalle, notaremos la cabeza demasiado larga en relación con el cuerpo y las patas. También, observaremos el desdoblamiento totalmente inhabitual en el arte del Paleolítico de algunas partes del contorno, en particular a nivel del pecho. Notaremos por fin la articulación muy angulosa entre la mandíbula y el cuello, que corta la parte anterior del cuello. Esta articulación angulosa es además una forma general de hacer de este artista, ya que la encontramos también en la articulación del vientre y de la pata trasera de los animales.
A Figura 4. Cueva de La Pasiega A: cierva roja (curvas grandes).

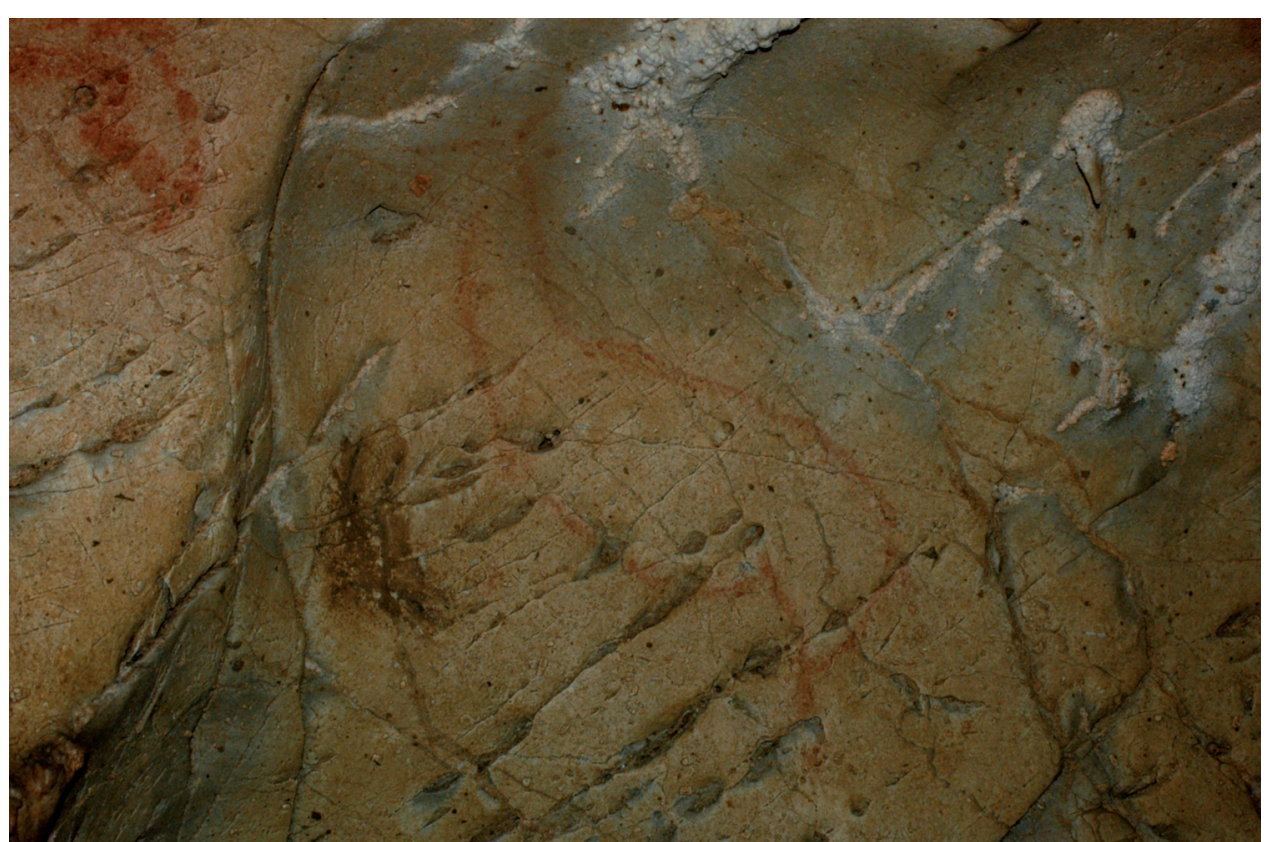

(- UNED. Espacio, Tiempo y Forma. Serie I, Nueva época. Prehistoria y Arqueología, t. 1, 2008. 


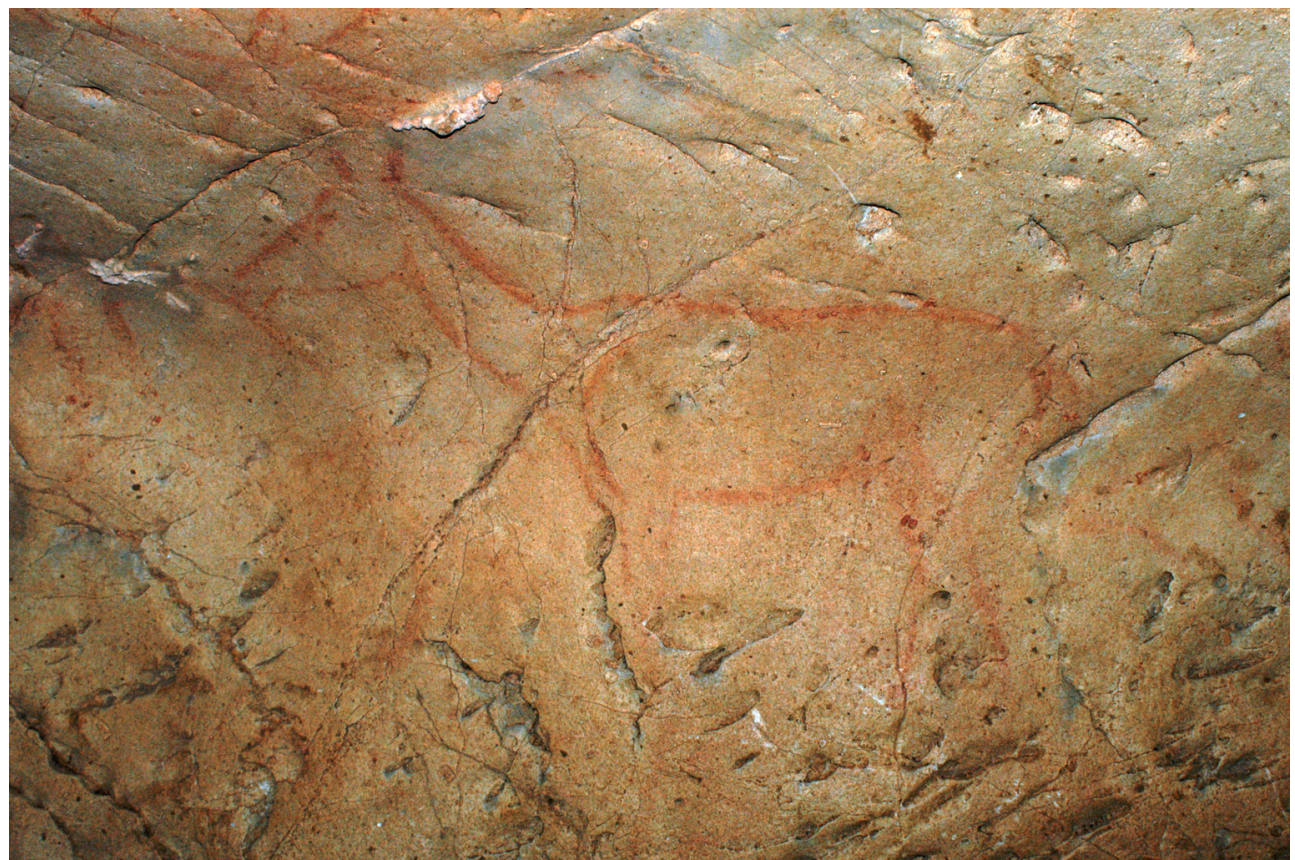

- Figura 5. Cueva de La Pasiega A: cierva roja (trazado orgánico).

\section{DE LA FIGURA AL SISTEMA ESTETICO}

La pared de la roca no tenía, para estos artistas, la función de mero soporte, como los paneles o los lienzos de nuestros pintores. Hemos intentado mostrar hasta qué punto las figuras se adaptaban a los relieves 0 a las fisuras naturales (Groenen, 2000). Esta intima conjunción de los dos llega hasta tal punto que los artistas no pensaron siempre que fuese necesario trazar completamente la figura, como lo podemos ver con un bisonte vertical de la cueva de Las Monedas, con la línea cervico-dorsal de un bisonte vertical (Ripoll Perelló, 1972: 16, n. ${ }^{\circ}$ 5, pl. 10, n. ${ }^{\circ}$ 2) o con el cuello y la cabeza de un caballo (Ripoll Perelló, 1972: 20, n. ${ }^{\circ}$ 30, pl. 32), por ejemplo. La técnica utilizada en ambos casos es evidentemente la del dibujo. Sin embargo, gracias a la recuperación juiciosa del relieve, estas dos obras tienen un modelado tal que aparecen como verdaderas esculturas. Es una característica importante de la mayor parte del arte parietal: se trata sobre todo de un arte de volumen, y no de un arte de superficie. Además sucede que la forma natural de la pared sugiera una parte importante del animal, como lo vemos con un bisonte rojo del "Panel de los Polícromos" de la cueva del Castillo (Alcalde del Río et al., 1912: fig. 122, n. ${ }^{\circ} 17$, pl. LXXI). El animal ya estaba presente en la pared y el artista sólo completó la parte que faltaba con un trazado rojo. Esta práctica es bastante frecuente: nos permite pensar que, para ciertas figuras por lo menos, lo único que el artista hizo fue revelar la criatura ya presente.

Así, la pared le da una materialidad a la figura animal, pero contribuye también a darle un espacio propio. Durante mucho tiempo, los prehistoriadores hicieron calcos sin preocuparse del soporte rocoso (Groenen, 1999). Ahora bien, parece que los animales no han sido trazados en cualquier lugar. Muy a menudo, la configuración de la figura es el resultado de un relieve o de una fisura. Sin duda alguna, el pequeño uro negro de la cueva del Castillo (Alcalde del Río et al., 1912: 145, fig. 134, nº 3, n. ${ }^{\circ}$ 6) está dispuesto en relación con las fisuras que sirven de línea del suelo para el animal. Lo mismo sucede evidentemente con un pequeño animal -canido o équido- de Las Monedas (Ripoll Perelló, 1972: 16, pl. XI-XII, n. ${ }^{\circ}$ 10) o con un mamut del Castillo (AIcalde del Río et al., 1912: 129, fig. 117, n. ${ }^{\circ}$ 76, pl. LXXIII). En todos estos casos, los animales no han sido colocados de cualquiera manera. No flotan en un espacio indeterminado, sino que están ubicados en un plano que les da una base. El concepto de espacio es además mucho más importante en el arte parietal de lo que podríamos pensar. Numerosas figuras de animales están efectivamente colocadas en escamas rocosas o insertadas en redes de fisuras que les ofrecen un marco, es decir, precisamente un espacio estructurado. El pequeño animal de Las Monedas, como hemos visto, no sólo fue dispuesto en un espacio delimitado por fisuras, sino también sobre una de estas fisuras, respetando además su oblicuidad (fig. 6). El hecho de ninguna manera es aislado, lo mismo ocurre con el uro y el mamut de la cueva del Castillo. Todas las figuras están dispuestas en una porción de espacio determinada por particularidades de la roca. Lógicamente, si el animal está dispuesto en un suelo y ubicado en un espacio cerrado, adquiere una materialidad, una corporeidad propia.

Esas diversas características le dan a la imagen paleolítica una coherencia formal extraordinaria, que la carga a la vez de una fuerza sugestiva y de una presencia casi hierática. Integración del volumen, figuración del movimiento, evo- 
cación de una línea del suelo para plantar el ser representado, definición de un espacio circunscrito para asegurarle un marco propio, todo está presente para que el animal surja en su realidad anatómica a través de su representación. La figura no se construye a partir de trazos simplemente yuxtapuestos. No se reduce pues en un encadenamiento trivial de trazos, pero se elabora, al contrario, a partir de un proyecto que pretende, gracias a sus trazos, producir un resultado óptico determinado, cuya forma y espacio son los dos puntos de arraigamiento conceptuales. Esa coherencia es evidentemente la expresión de un sistema estético, como la talla laminaria es la manifestación de un sistema técnico coherente, y no el producto trivial de una sucesión de levantamientos.

El periodo durante el cual los artistas paleolíticos decoraron las paredes de las cuevas es evidentemente demasiado largo y los grupos demasiado numerosos para poder pensar que este arte parietal sea el resultado de un sistema estético único. En la ausencia de una cronología fina, nos es difícil tratarlo de otro modo que como una producción atemporal. De igual forma, la pobreza de lo que nos llegó en ciertas zonas nos impide actualmente imaginar un análisis espacial de este fenómeno. Al contrario, es posible aprehender diferencias entre las obras de una misma red. Es el caso para numerosas figuras animales finamente grabadas, a menudo de medidas pequeñas y ejecutadas sobre una parte plana de la pared. El pequeño caballo de La Pasiega (Balbín Behrmann \&t González Sáinz, 1994: 273, fig. 4, n. D2/6), por ejemplo, ilustra bien esta manera diferente de pensar la figura. El tamaño del animal no excede $26 \mathrm{~cm}$ de largo y $14 \mathrm{~cm}$ de altura a la cruz. Fue realizado con un trazado único, continuo, flexible y poco profundo. Buscariamos en vano esta "puesta en forma" tan característica del arte de las cuevas. El campo operatorio toma aquí el papel de un soporte sencillo sobre el cual aparece la representación, ya que se trata en efecto de una representación. La imagen no se da de manera "monolítica", y tampoco pretende dar a la figura esta impresión de presencia. En cambio, se esmera en reproducir el animal con la sugestión gráfica - podríamos decir, con Gombrich, con la ilusión- del expresado de las formas y de los volúmenes anatómicos.

Esta manera de proceder evoca numerosos dibujos o grabados de épocas históricas, en los cuales los trazos, a menudo múltiples, están dispuestos hábilmente para creer la ilusión de la forma, del volumen o del movimiento. Siempre en la red B de La Pasiega, un pequeño caballo, de unos veinte centímetros, comprende la cabeza, el cuello, las dos patas delanteras y parte de las líneas del vientre y del dorso (Balbín Behrmann \& González Sáinz, 1996: 286-287, fig. 8, n.. B.7/33). El contorno fue ejecutado por trazos grabados finos, múltiples y relativamente cortos, que le dan una línea nerviosa, de ejecución progresiva. Otra figura de esta red presente los cuartos delanteros de una cierva (Balbín Behrmann

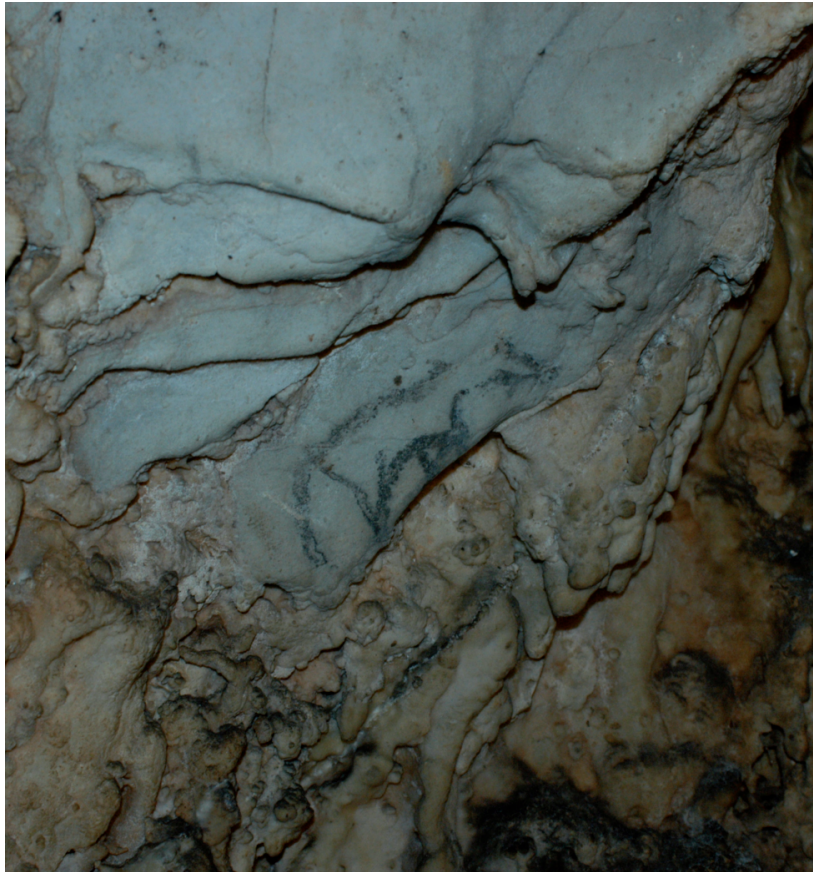

A Figura 6. Cueva de Las Monedas: animal negro con línea de suelo.

Ct González Sáinz, 1996: 288, fig. 10, n. ${ }^{\circ}$ B.7/35), ejecutada con la misma técnica: los trazos finos múltiples se encadenan en segmentos de trazos más o menos paralelos. No es ininteresante notar la acumulación de incisiones al nivel de la barbada y del cuello del animal. El procedimiento es evidentemente destinado a dar la ilusión del modelado anatómico y fue abundantemente empleado en las representaciones de "ciervas rascadas" ejecutadas en las paredes de las cuevas cantábricas como El Castillo (Alcalde del Río et al., 1911: 170-174, fig. 166-169) o Altamira (Breuil \&t Obermaier, 1984: 95 et 105 , fig. 58 et 68 ).

En conclusión, como los artistas de los periodos históricos, los pintores y grabados de la prehistoria paleolítica poseían una serie de técnicas tremendamente rica, pero también un saber hacer experimentado. Este dominio les permitió llenar sus producciones estéticas con una presencia extraordinariamente fuerte. Es sorprendente en efecto constatar hasta qué punto los motivos figurativos imponen su unidad y su coherencia formal. Éstos nunca parecen como la mera adición de trazos independientes, pero cada uno de ellos se da, al contrario, desde el resultado final. Mejor, la figura animal nunca es desencarnada. Adquiere muy a menudo su forma, su volumen -su realidad, deberíamos decirgracias a la integración de particularidades naturales o de procedimientos gráficos sugestivos. Pues el arte parietal es mucho más un arte de la sugestión que un arte de la ilusión. Lo que nos parece, en todo caso, cierto es que esta producción estética procede de profesionales y en ningún caso de diletantes. 


\section{BIBLIOGRAFÍA}

Alcalde del Río H., Breuil H. y L. Sierra, 1912. Les cavernes de la Région cantabrique, Monaco, Vve A. Chêne, 265 p., 258 fig., 100 pl.

AltunA J., 1997. L'art des cavernes en pays basque. Les grottes d'Ekain et Altxerri, Paris, Le Seuil, 200 p., 211 fig. (coll. "Arts rupestres").

Balbín Behrmann R. de y González Sáinz C., 1994. Un nuevo conjunto de representaciones en el sector D.2 de la cueva de La Pasiega (Puente Viesgo, Cantabria), en: J.A. Lasheras (ed.), Homenaje al Dr. Joaquín González Echegaray, Madrid, Ministerio de Cultura, pp. 269-280, 8 fig., 8 ill. col. ("Museo y Centro de Investigación de Altamira", Monografias no. 17).

Balbín Behrmann R. de y GonzÁlez Sáinz C., 1996. Las pinturas y grabados del corredor B.7 de la cueva de La Pasiega (Cantabria), en: A. Moure Romanillo (ed.), "El hombre fósil". 80 años después. Volumen conmemorativo del 50 aniversario de la muerte de Hugo Obermaier, Santander, Universidad de Cantabria, pp. 271-294, 10 fig., $5 \mathrm{pl}$.

Breuil H. y Obermaier H., 1984 [réimpr. de 1935]. La Cueva de Altamira en Santillana del Mar, Madrid, El Viso, 293 p., 183 fig., 52 pl.

Clottes J., Menu M. Y Walter P., 1990. La préparation des peintures magdaléniennes des cavernes ariégeoises, en: Bulletin de la Société préhistorique française, 87, pp. 170-192.

CRÉMADĖs M., 1992. Nouvelle lecture d'objets d'art mobilier paléolithique de la collection Piette (MAN), en: Bulletin de la Société préhistorique de l'Ariège, 47, pp. 107-131.

CRÉMADĖs M., 1996. L'art mobilier pyrénéen: analogies technologiques et relations inter-sites, en: H. Delporte y J. Clottes (dir.), Pyrénées préhistoriques. Arts et sociétés. Actes du 118e Congrès national des Société savantes (Pau, 1993), Paris, C.T.H.S., pp. 367-380.

FRITZ C., 1999. La gravure dans l'art mobilier magdalénien, du geste à la représentation. Contribution de l'analyse microscopique, Paris, Maison des sciences de l'Homme, 216 p., 185 fig. ("D.A.F., no. 75").
Groenen M., 1994. Pour une histoire de la préhistoire. Le Paléolithique Grenoble, Jérôme Millon, 603 p., 99 ill., 24 tabl. (coll. "L'Homme des Origines").

Groenen M. (1999). Du relevé de la représentation à la lecture du dispositif pariétal, en: Anthropologie et préhistoire, 110, pp. 3-23.

Groenen M. (2000). Sombra y luz en el arte paleolítico, Barcelona, Ariel, $135 \mathrm{p}$.

GROENEN M., 2007a. Voir l'image préhistorique: bilan des travaux dans la grotte ornée d'El Castillo (Cantabrie, Espagne), en: J. Évin (dir.), Un siècle de construction du discours scientifique en préhistoire, vol. III. "...aux conceptions d'aujourd'huin. Actes du Congrès du Centenaire de la Société préhistorique française (Avignon, 21-25 septembre 2004), Paris, S.P.F., pp. 307-321, 16 fig. (XXVle Congrès préhistorique de France).

Groenen M., 2007b. Principios de lectura del arte parietal en las cuevas decoradas del Monte del Castillo. En: J. M. Maillo y E. Baquedano (ed.), Victoria Cabrera. Obra selecta. Miscelánea en Homenaje a Victoria Cabrera, Madrid, UNED y Museo Arqueológica Regional, pp. 42-53.

Groenen M. y Martens D. (en prensa). Nouvelles attributions dans les grottes du Monte del Castillo (Puente Viesgo, Cantabrie). Communication présentée dans le cadre du 15e Congrès de l'Union Internationale des Sciences Préhistoriques et Protohistoriques (UISPP, Lisbonne, 4-10 septembre 2006).

Lerol-Gourhan A., 1978. Préhistoire de l'art occidental, Paris, Mazenod, 482 p., 804 fig.

RICHARD E., 2005-2006. Étude des techniques de peinture du "Recoin des Tectiformes" de la grotte d'El Castillo (Cantabrie, Espagne). Mémoire de licence (inédit), Bruxelles, Université Libre de Bruxelles, 2 vol.

Ripoll Perelló E. (1972). La cueva de Las Monedas en Puente Viesgo (Santander), Barcelona, Diputación Provincial de Barcelona/Instituto de Prehistoria y Archeología, 67 p., 30 fig., 35 pl. ("Monografías de 